\title{
STUDIES OF NARROW EMISSION LINES IN AGNs
}

\author{
Alexei V. Filippenko \\ Department of Astronomy \\ University of California \\ Berkeley, CA 94720 \\ USA
}

\begin{abstract}
Optical spectra having moderately high resolution $(\sim 2-5 \AA)$ are being used to study the profiles of narrow emission lines in active galactic nuclei (AGNs). It is often found that forbidden lines associated with high critical densities for collisional deexcitation are the broadest. A good example is [O III] $\lambda 4363\left[n_{e}\right.$ (crit) $\left.\approx 3 \times 10^{7} \mathrm{~cm}^{-3}\right]$, whose width can be more than twice that of [O III] $\lambda 5007\left[n_{e}\right.$ (crit) $\left.\approx 8 \times 10^{5} \mathrm{~cm}^{-3}\right]$. The tight correlation between line width and $n_{e}$ (crit) implies that a much larger range of densities $\left(\sim 10^{2}-10^{7} \mathrm{~cm}^{-3}\right)$ must be present among clouds in the narrow-line region than was previously believed. At times there almost appears to be a continuity between the narrow- and broadline regions. In some objects the dense, high-velocity clouds are optically thick to ionizing radiation, since they emit [O I] $\lambda 6300$ as well as species of much higher ionization (such as $[\mathrm{Ne} \mathrm{V}] \lambda 3426$ ). These results help eliminate several difficulties in photoionization models of LINERs. It may also be possible to use the observed line widths as probes of the gravitational potential in AGNs.
\end{abstract}

\section{INTRODUCTION}

In the standard picture of quasars and Seyfert galaxies, radiation produced by a "central engine" is thought to photoionize gas located in two rather distinct volumes (see, e.g., Weedman 1977). Clouds in the broad-line region (BLR) live $\sim 0.1-1 \mathrm{pc}$ from the nucleus, are quite dense $\left(n_{e} \approx 10^{8}-10^{10} \mathrm{~cm}^{-3}\right)$, and have characteristic velocities of $\sim 3000-10000 \mathrm{~km} \mathrm{~s}^{-1}$; the corresponding parameters for the narrow-line region (NLR) are $\sim 10-500 \mathrm{pc}, \sim 10^{3}-10^{4} \mathrm{~cm}^{-3}$, and $\sim 300-1000 \mathrm{~km} \mathrm{~s}^{-1}$. The temperature of the ionized gas, determined primarily by the equilibrium between photoionization heating and radiative cooling, is $~$ $10000-20000 \mathrm{~K}$ in both regions.

The emission lines in AGNs often have nearly symmetrical profiles. When observed at spectral resolutions of $\sim 10 \AA$, those from the NLR are well described by single Gaussians, and in a given object the widths of different lines are similar (Koski 1978). Hence, photoionization models have generally dealt only with the total intensities of individual lines (e.g., Davidson and Netzer 1979). The results have been encouraging, but important difficulties remain. 
It has recently become clear that this simple picture has serious shortcomings, and that any quantitative model based on it can provide at best a rough physical description of AGNs. Osterbrock (1979), for example, has stressed that in certain objects there is a range in density among the narrow-line clouds $\left(n_{e} \approx 10^{3}-10^{6} \mathrm{~cm}^{-3}\right.$ ). There is increasing evidence that several distinct families of clouds exist in the BLR, and that $n_{e} \gtrsim 10^{11} \mathrm{~cm}^{-3}$ in at least some of the gas (Puetter 1986). Moreover, many broad emission lines exhibit complex profiles. In this paper I will show that the profiles of narrow emission lines can also be used to impose constraints on realistic models.

\section{A CLOSE LOOK AT THE NLR}

Optical spectra having moderately high resolution $(\sim 2-5 \AA)$ were obtained with an Intensified Reticon on the 2.5-m du Pont telescope at Las Campanas Observatory (Chile) in order to study the narrow emission lines in several AGNs. These are illustrated in Filippenko and Halpern (1984; FH84) and in Filippenko (1985; F85).

One object of particular interest is MR 2251-178, the first QSO to initially be identified as a result of $\mathrm{X}$-ray observations. Its spectrum is characterized by very broad permitted lines and considerably narrower forbidden lines (and permitted-line cores) superposed on a strong nonstellar continuum. The lines are undoubtedly produced by photoionized gas. On the other hand, the intensity ratio $(R)$ of [O III] $\lambda \lambda 4959+5007$ to [O III] $\lambda 4363$ is only $\sim 10$, far lower than normal in AGNs. If $n_{e} \lesssim 10^{4} \mathrm{~cm}^{-3}$, this implies that $T_{e} \gtrsim 10^{5} \mathrm{~K}$, which is much too high for the $\mathrm{O}^{++}$region. Osterbrock, Koski, and Phillips (1976) recognized this problem in several broad-line radio galaxies, and suggested that $n_{e} \approx 10^{6}$ $\mathrm{cm}^{-3}$ in clouds which produce the [O III] emission.

Direct evidence that high densities do indeed resolve the problem in MR 2251-178 is found from a comparison of the line profiles. As shown in Figure 9 of F85, there is a wide range of line widths and profile shapes, indicating that clouds having different physical properties are present in the NLR. In particular, lines associated with high critical densities $\left[n_{e}(\right.$ crit $\left.)\right]$ are broader than those with low values of $n_{e}$ (crit), so they must be formed in the denser, more rapidly-moving clouds. [O III] $\lambda 4363\left[n_{e}\right.$ (crit) $\left.\approx 3 \times 10^{7} \mathrm{~cm}^{-3}\right]$, for example, is much broader than [O III] $\lambda 5007\left[n_{e}(\mathrm{crit}) \approx 8 \times 10^{5} \mathrm{~cm}^{-3}\right.$ ]; line width is therefore not correlated with ionization potential $(\chi)$, unlike the case in some AGNs (De Robertis and Osterbrock 1984). Note that a large range of densities would not necessarily be excluded if the correlation between line width and $\chi$ were better than that between width and $n_{e}$ (crit), but an unambiguous conclusion would then be difficult to reach without a considerably more detailed analysis.

Since the [O III] lines in MR 2251-178 have such different profiles, it is of interest to examine the ratio $R$ as a function of velocity (i.e., position in the line profile). If the gas is photoionized by ultraviolet radiation from the nucleus, then $T_{e} \approx 15000 \mathrm{~K}$ in the $\mathrm{O}^{++}$zone, and the changing value of $R$ directly demonstrates that high-velocity clouds $\left(v \gtrsim 1000 \mathrm{~km} \mathrm{~s}^{-1}\right.$ ) have $n_{e} \gtrsim 5 \times 10^{6} \mathrm{~cm}^{-3}$ (see Fig. 10 in F 85). This suggests that an "intermediate" region exists between the NLR and BLR.

The same results are found in Pictor $\mathbf{A}$, a broad-line radio galaxy with strong forbidden lines spanning a wide range of ionization states. As shown in 
Figure 1, there is an excellent correlation between line width and $n_{e}$ (crit). Since line emission per unit mass of gas increases linearly with $n_{e}$ below $n_{e}$ (crit), and remains constant above it, different forbidden lines act as effective tracers of $n_{e}$ when there is a sufficiently large range of densities among the clouds. This is precisely what must be happening in the NLR of Pictor A. In fact, it is known from the [S II] $\lambda \lambda 6716,6731$ doublet that clouds having $n_{e} \lesssim 500 \mathrm{~cm}^{-3}$ are present in the NLR, yet the [O III] ratio $R$ indicates that densities in excess of $10^{6}$ or even $10^{7} \mathrm{~cm}^{-3}$ must also exist (F85). Once again, the highest densities are only an order of magnitude or two below those in the BLR, and the lines produced in the "intermediate" region are quite broad. Similar conclusions have been drawn by Carswell et al. (1984).

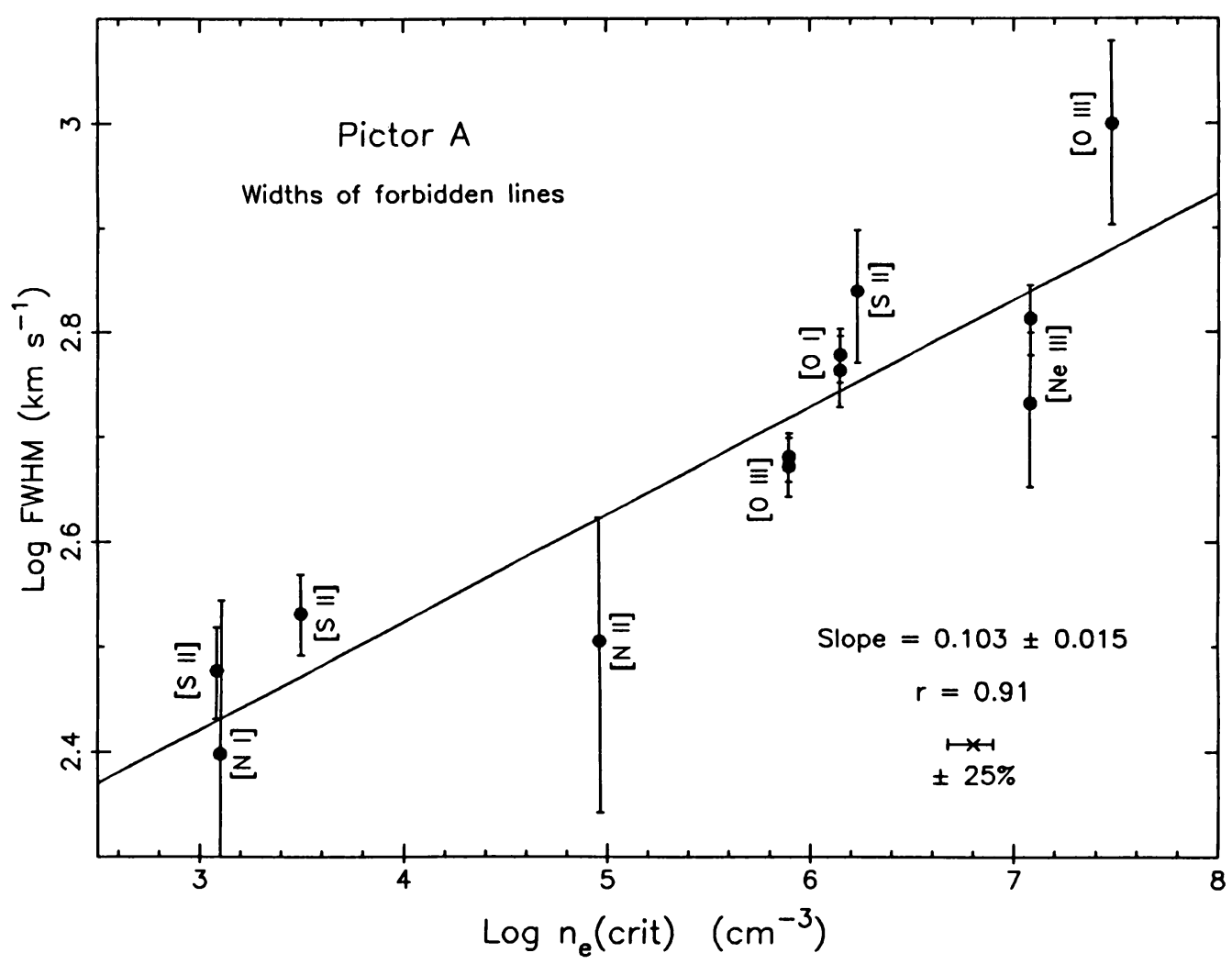

Figure 1: Log FWHM is plotted against $\log n_{e}$ (crit) for 12 forbidden lines in Pictor A. An error bar of $\pm 25 \%$ indicates probable uncertainties in theoretical calculations of $n_{e}$ (crit). A linear least-squares fit to the points is shown; the correlation coefficient is 0.91 , and the probability that the two variables are uncorrelated is virtually zero. Clouds in the NLR must therefore span a very large range of densities. 
The correlation between line width and $n_{e}$ (crit) may be useful as a probe of the gravitational potential in AGNs (FH84; Filippenko and Sargent 1986). If clouds in the NLR move around the nucleus in orbits of the form $v \propto r^{\beta}$, and if the ionizing flux from the central engine is constant over a dynamical time scale, then $v \propto(n U)^{-\beta / 2}$, where $U$ is the ionization parameter $\left(U \propto L n^{-1} r^{-2}\right)$. Under the assumption of Keplerian orbits, and if $U$ is independent of distance from the nucleus, this becomes $v \propto n^{0.25}$, which is close to the observed relation $\left(v \propto n^{0.2}\right)$ in the Seyfert 1 galaxy NGC 7213 (FH84). If the potential is logarithmic, on the other hand, no differences in line width are expected. Many other conditions are also possible, of course.

\section{THE IONIZATION MECHANISM IN LINERs}

Filippenko and Sargent (1985) and F85 have shown that the range of densities found in the NLR of MR 2251-178, NGC 7213, and Pictor A is also present in many "low-ionization nuclear emission-line regions" (LINERs; Heckman 1980). The strengths of transauroral and auroral lines (e.g., [O III] $\lambda 4363$ ) therefore tend to be enhanced relative to the corresponding nebular lines ([O III $] \lambda \lambda 4959$, 5007), and the emitted spectra can strongly resemble those produced by shockheated gas. In fact, heating by shocks was originally thought to be the ionization mechanism in LINERs (e.g., Koski and Osterbrock 1976; Fosbury et al. 1978), but modern photoionization models (Ferland and Netzer 1983; Halpern and Steiner 1983) are more successful at reproducing the observed spectra, especially when the great range of densities is properly taken into account (Péquignot 1984).

One way in which the models have been modified is that large optical depths are now included in the NLR. The presence of $[\mathrm{O} \mathrm{I}] \lambda 6300$ with width comparable to that of [O III] $\lambda 5007$ in Pictor A and other objects (FH84; F85) demonstrates that the densest clouds $\left(n_{e} \gtrsim 10^{6} \mathrm{~cm}^{-3}\right)$ must in some cases be optically thick to ionizing radiation. Soft X-rays, however, are able to penetrate deep into these clouds, ionizing roughly $10 \%$ of the hydrogen atoms in vast regions (see, e.g., Halpern 1982). The great strength of [O I] $\lambda 6300$ and certain other lines is then easily explained, because they are best produced in partially-ionized gas of just this type.

\section{ACKNOWLEDGMENTS}

I would like to thank the night and day crews at Las Campanas Observatory for their help. The figure in this paper is reproduced courtesy of The Astrophysical Journal. Much financial assistance was provided by the Miller Institute for Basic Research in Science (U.C.B.), as well as by the organizing committee of IAU Symposium 119.

\section{REFERENCES}

Carswell, R. F., Baldwin, J. A., Atwood, B., and Phillips, M. M. 1984, Ap. J., 286, 464.

Davidson, K., and Netzer, H. 1979, Rev. Mod. Phys., 51, 715. 
De Robertis, M. M., and Osterbrock, D. E. 1984, Ap. J., 286, 171.

Filippenko, A. V. 1985, Ap. J., 289, 475 (F85).

Filippenko, A. V., and Halpern, J. P. 1984, Ap. J., 285, 458 (FH84).

Filippenko, A. V., and Sargent, W. L. W. 1985, Ap. J. Suppl., 57, 503.

Filippenko, A. V., and Sargent, W. L. W. 1986, in Structure and Evolution of Active Galactic Nuclei, ed. G. Giuricin et al. (Dordrecht: Reidel).

Fosbury, R. A. E., Mebold, U., Goss, W. M., and Dopita, M. A. 1978, M. N. $R$. A. S., 183, 549 .

Halpern, J. P. 1982, Ph.D. thesis, Harvard University.

Halpern, J. P., and Steiner, J. E. 1983, Ap. J. (Letters), 269, L37.

Heckman, T. M. 1980, Astr. Ap., 87, 152.

Koski, A. T. 1978, Ap. J., 223, 56.

Koski, A. T., and Osterbrock, D. E. 1976, Ap. J. (Letters), 203, L49.

Osterbrock, D. E. 1979, in Active Galactic Nuclei, ed. C. Hazard and S. Mitton (Cambridge: Cambridge University Press), p. 25.

Osterbrock, D. E., Koski, A. T., and Phillips, M. M.·1976, Ap. J., 206, 898.

Péquignot, D. 1984, Astr. Ap., 131, 159.

Puetter, R. C. 1986, these proceedings.

Weedman, D. W. 1977, Ann. Rev. Astr. Ap., 15, 69.

\section{DISCUSSION}

Burbidge : Why do you suppose that the clouds are bound?

Filippenko : The clouds themselves are almost certainly not self-gravitating bound systems, if that is what you are asking. It may be true, however, that the clouds are bound to the nucleus; if so, one can study the gravitational potential by examining the line widths. Of course, many other effects e.g. radiative acceleration may contribute to the line widths, and in this case my model is too simplistic. It was just presented as an example.

Wandel : The cloud kinematics in the [OIII] emission region may be dominated by the gravity of the galactic nucleus, as suggested by the correlation between [OIII] line width and stellar velocity dispersion (Heckman and Wilson 1984), while the (narrow) lines of higher critical density, which are supposedly emitted from smaller radii, may have profiles due to different kinematics. Is there a qualitative difference in the profiles of those line (except from just being broader), which could suggest a different kinematic setting ?

Filippenko : This is difficult to address observationally, since different portions of a line profile are produced by different regions, but one cannot easily determine the relative contributions. [OI] 6300 is a good example of this. Moreover, the signal-to-noise ratios in the lines of highest critical density (e.g., [Ne V]) are not sufficiently high. I will look into this question more thoroughly in future studies.

Petrosian : It appears that the range of densities in narrow line regions 
and broad line regions, are beginning to overlap. Is it, therefore, still useful to think about two distinct regions or should one consider a continuum for cloud densities and velocities for the whole line emitting region.

Filippenko : I believe the answer depends on the types of questions that are being addressed, and on the particular objects under consideration. In general, the classification into a BLR and a NLR is still a useful one, especially since some active galaxies show no evidence for a BLR at all. Moreover, in certain objects there appears to be much less emission from the intermediate-density region than from the low-density and highdensity regions. There is increasing evidence, however, that a real continuity in density and velocity exists between the NLR and BLR in many AGNs, and in these one must take the data seriously when constructing models.

Dultzin-Hacyan : With respect to the separation between BLR and NLR, is it not a bit artificial in view of what has been said, and also taking into account the reports by Drs. van Groningen and $\mathrm{K}$. Meyers on observed broad [OIII] lines?

Filippenko : Let me once again emphasize that a main conclusion from my studies is that in many objects there is indeed a continuity in density and line width, as opposed to discrete BLRs and NLRs. The work you refer to supports this view.

McAdam : The presence of dense clouds in the narrow line region is necessary for any intrinsic absorption mechanism for low frequency variability in quasars - what range of collision frequencies and covering factors do you think are present in your sources?

Filippenko : The covering factors are probably in the range 0.01-0.1. Collision frequencies of particles in the clouds are roughly $10^{-3}$ to 1 , depending on the density.

Alloin : In the NLR of NGC 3783, we found this relationship between the critical density and the line width, by studying, through gaussian decompositions, profiles in details for lines of different excitations from [OII] up to [FeX]. Indeed, when one goes to lines of higher excitation and larger critical density, one requires the addition of a larger blue-shifted component. This can be understood in terms of an increase towards the centre, both of the density in the NLR filaments and of the mean "turbulent" velocity which governs the line-width, as well as of a radial velocity component.

I wish to emphasize the fact that it might be time, now, to perform a more detailed confrontation between photoionized models and line intensities in the NLR, considering the line ratios within a given slab of material in the velocity space, rather than the integrated ones.

Filippenko : I agree whole heartedly. 